
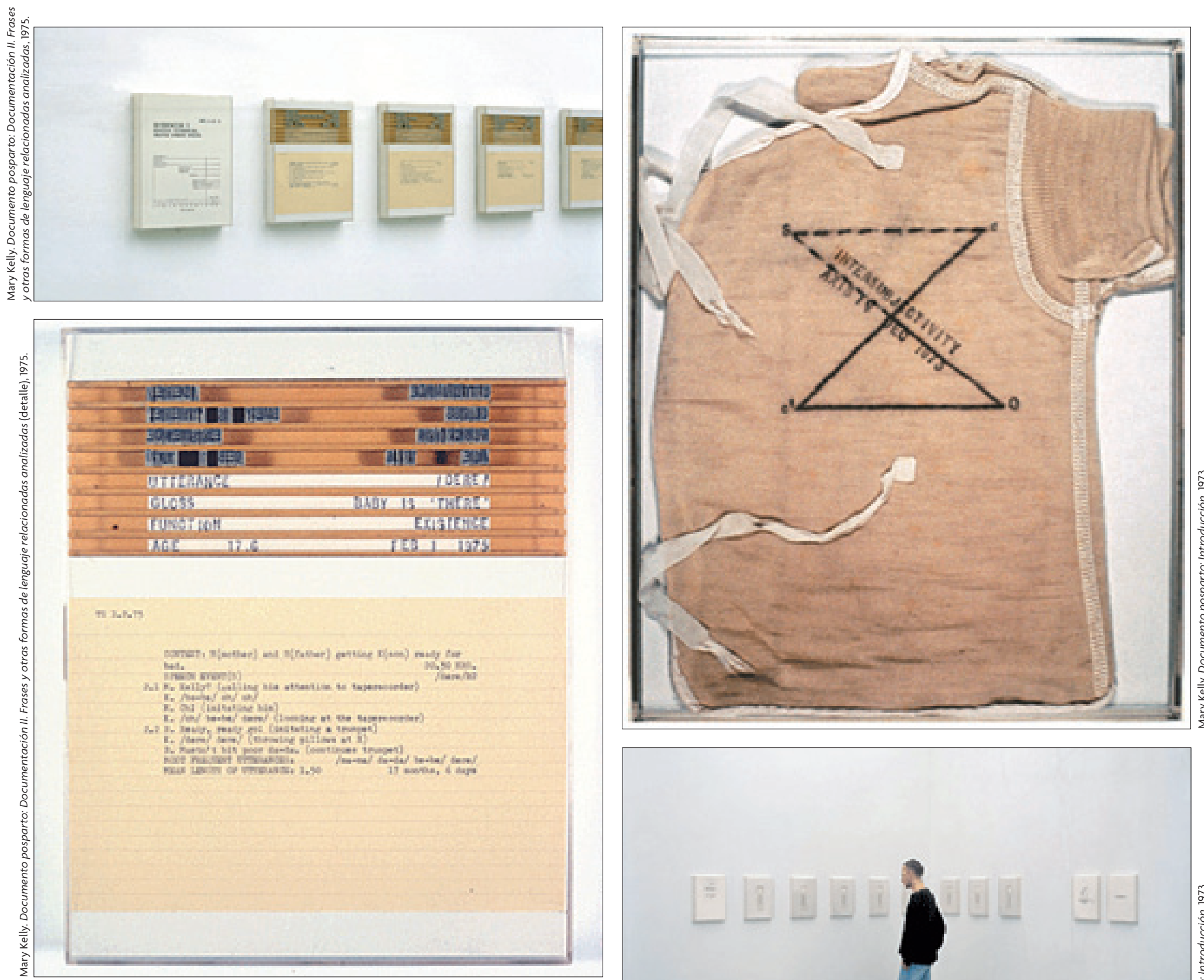

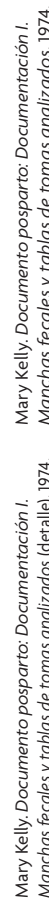

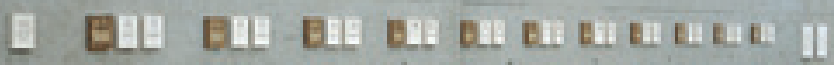
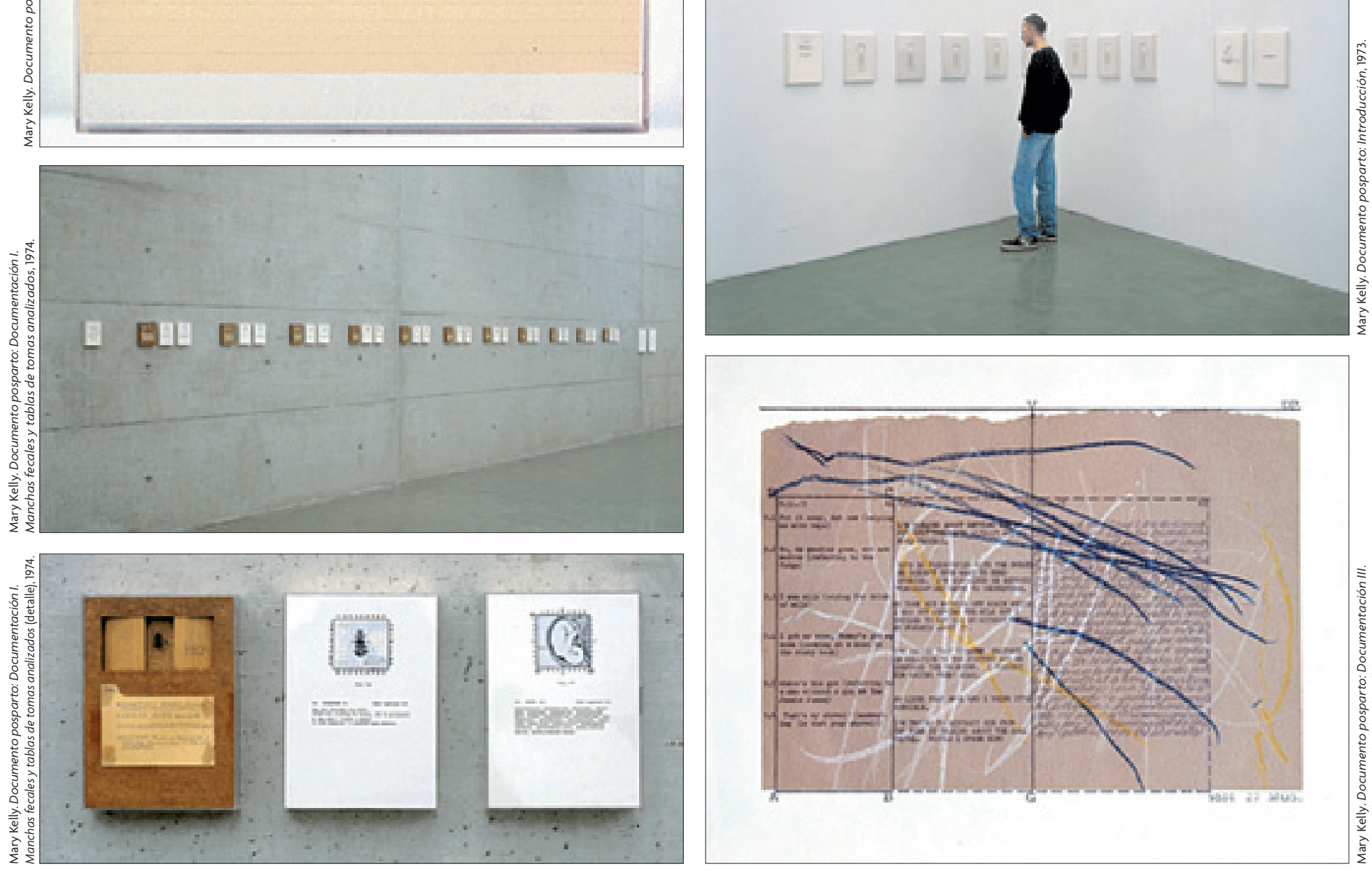


\title{
LA REPRODUCCIÓN EN LA VIDA DE LAS MUJERES APORTACIONES DE LA ANTROPOLOGÍA
}

\author{
Maribel BlázQuez Rodríguez y Ma Jesús Montes Muñoz
}

La antropología social y cultural ha estudiado la reproducción desde el punto de vista de diversas especialidades - parentesco, simbolismo, feminismo, salud-, y ha señalado tanto las dimensiones que abarca como el carácter social, construido y central para el mantenimiento de una sociedad. Esta mirada permite a las autoras sugerir ciertas hipótesis a partir de las cuales entender la reproducción como un asunto vertebrador de la vida de las mujeres en la actualidad, que debería ser objeto de estudio relevante para la ciencia.

Palabras clave: mujeres, reproducción, antropología, género.

En este artículo quisiéramos acercarnos a uno de los temas que desafía hoy la vida de todas las mujeres, la reproducción; y ésta, tomada en consideración desde la perspectiva iniciada y desarrollada por la antropología. Para empezar, debemos precisar de qué hablamos cuando utilizamos el término reproducción; ya que, como acertadamente subrayaron Olivia Harris y Kate Young (1981; en Comas, 1998), este concepto engloba diferentes dimensiones que, aun cuando guarden relación, son bien dispares y poseen significados bien distintos. En este sentido, es necesario distinguir: la reproducción humana o biológica, la reproducción del trabajo y la reproducción social. Veamos brevemente cada una de ellas: la primera, la reproducción biológica o procreación, se refiere al hecho de aumentar el número de individuos de un grupo social; la segunda implica la reproducción del esquema de división sexual en el mundo del trabajo, por cuanto se asignan actividades diferentes a hombres y mujeres, lo que perpetúa la diferencia. Y la tercera, la reproducción social o sistémica, se refiere a la que transmite y da continuidad a un determinado sistema social repitiendo ideas, valores, normas, así como estableciendo la organización del parentesco, de la economía y de cualquier otro ámbito.

Tener en cuenta estas dimensiones de la reproducción permite interrogarnos acerca de cuáles son sus dinámi- cas y la problemática actual que presenta, con su incidencia especial en la vida de las mujeres. Baste como ejemplo la situación particular de aquellas que se dedican a la ciencia, para quienes las demandas y exigencias de la reproducción biológica interfieren en su carrera académica y profesional; así, se ven obligadas a adaptar esta última a la primera con el fin de hacerlas compatibles, si no es que tienen que renunciar a una de ellas en favor de la otra. Consideramos que abordar adecuadamente el entramado de la reproducción se torna imprescindible para promover eficazmente el avance de las mujeres en todas las áreas sociales; además, es de esperar que el conocimiento del complejo fenómeno de la reproducción contribuya a hacer visibles las desigualdades aún presentes en nuestra sociedad. En definitiva, se trata de proponer el estudio de un tema que afecta específicamente a la condición femenina, aunque también a los varones, y que merece en consecuencia el esfuerzo de detenernos en él para repensarlo.

Con este fin, en un primer apartado de este artículo repasaremos algunas de las aportaciones de los campos de la antropología interesados en la reproducción $\mathrm{y}$, a continuación, expondremos nuestros argumentos acerca de la centralidad que la reproducción ocupa todavía hoy en la vida de las mujeres planteando diversos ejemplos. 


\section{LAS APROXIMACIONES A LA REPRODUCCIÓN}

Dentro del campo de la antropología, encontramos tempranas incursiones en la cuestión de la reproducción biológica. En algunas etnografías clásicas, como las de Bronislaw Malinowsky y Ashley Montagu (en Browner y Sargent, 1990: 221), se describen los aspectos socioculturales más relevantes, como las normas, los tabúes y los valores en torno a la menstruación, el embarazo, el parto y el periodo posterior al nacimiento. En una línea similar, aunque más cercana y centrada en el territorio del Estado español, destaca la recogida de datos que ofrece la Encuesta del Ateneo de 1901-1902 (Limón y Castellote, 1980) sobre costumbres relacionadas con algunas fases del ciclo vital: nacimiento, matrimonio y muerte. En dicha encuesta, se plasman también las creencias, las supersticiones, incluso los «remedios» con los que los grupos sociales manejaban todo el proceso de la gestación, y en los que se muestran los roles, las normas y las expectativas establecidas para las mujeres y los hombres en esta etapa de la reproducción biológica. Se trata de una etapa crucial en la vida de los individuos y en el ordenamiento social, pues, como planteó Arnold van Gennep, el nacimiento de un hijo debe entenderse como un «rito de paso» que da lugar al cambio de estatus de los progenitores (de mujer a madre y de hombre a padre), al mismo tiempo que se reconstituyen los vínculos de parentesco.

A partir de los años setenta, surgen un conjunto de etnografías (Blázquez, 2005) dirigidas a mostrar cómo la procreación, aun cuando constituya un proceso biológico, no puede ser entendida al margen de las relaciones sociales ni de los sentidos y las implicaciones que suponen para cada grupo social en cada contexto histórico. La reproducción no solo es un puente entre lo biológico y lo cultural, sino que articula los roles de género -lo concerniente a la feminidad y la maternidad, la masculinidad y la paternidad- dentro de un sistema ideológico y sociopolítico (Browner y Sargent, 1990). Estos estudios introducen un análisis feminista y de clase social que vincula la reproducción biológica con las otras dimensiones, tales como la división sexual del trabajo y el mantenimiento de un determinado sistema social patriarcal y capitalista.

Las interrelaciones entre ambos niveles (lo biológico con lo sociocultural) indican que el cuerpo de la mujer no es solo una entidad consistente en un sustrato biológico, sino que sobre él actúan conceptualizaciones bien definidas, es decir, formas concretas de atenderlo y vivirlo que descansan, a su vez, sobre unas matrices políticas y socioculturales. Algunos trabajos etnográficos sobre el tema que se focalizan en la fertilidad, el proceso del parto, el embarazo, la lactancia... muestran cómo todos estos procesos corporales femeninos son social y culturalmente regulados (desde los modelos de atención biomédica hasta los rituales y tabúes).

En el Estado español disponemos de diversos estudios de antropología de la salud acerca de las ideas, las normas y los roles que se asignan a las mujeres a partir de la procreación. Tales instancias proceden particularmente del ámbito sanitario -que podríamos definir como un «biopoder» en el sentido foucaultiano-, el cual configura tipos de normatividad y formas de subjetividad que, entre otras cuestiones, colocan el cuidado y la maternidad en el epicentro de la vida de las mujeres, tal como lo plantea Mari Luz Esteban et al. (2010).

Desde otros campos como la antropología del parentesco - preocupada por la formulación de las ideas, representaciones, normas y usos relativos a los procesos de procreación, adscripción y crianza (Fons et al., 2010)-, se analizan los cambios actuales en las formas de familia debidas a las migraciones, adopciones y nuevas tecnologías reproductivas, desvelando algunos nuevos sentidos para esta.

Estos campos especializados de la antropología se han desarrollado de una forma independiente $y$ con marcos teóricos diversos; sin embargo, al coincidir en un mismo objeto de estudio, han venido a

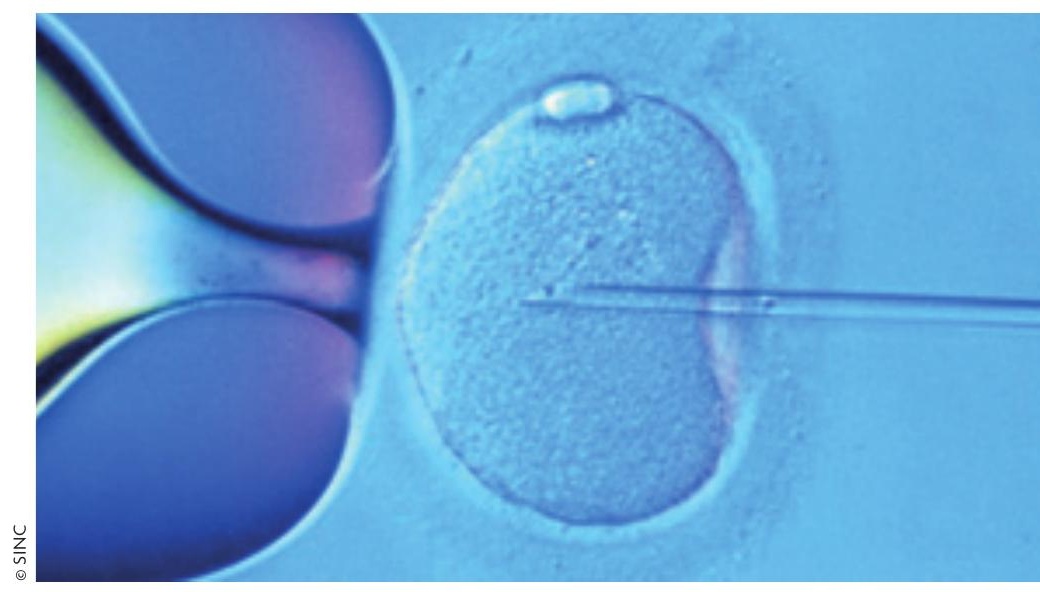

Hoy en día, las mujeres tienen la libertad de poder decidir qué opción de procreación encaja más con sus deseos: un claro ejemplo son las «madres por elección», antes llamadas «madres solteras». 


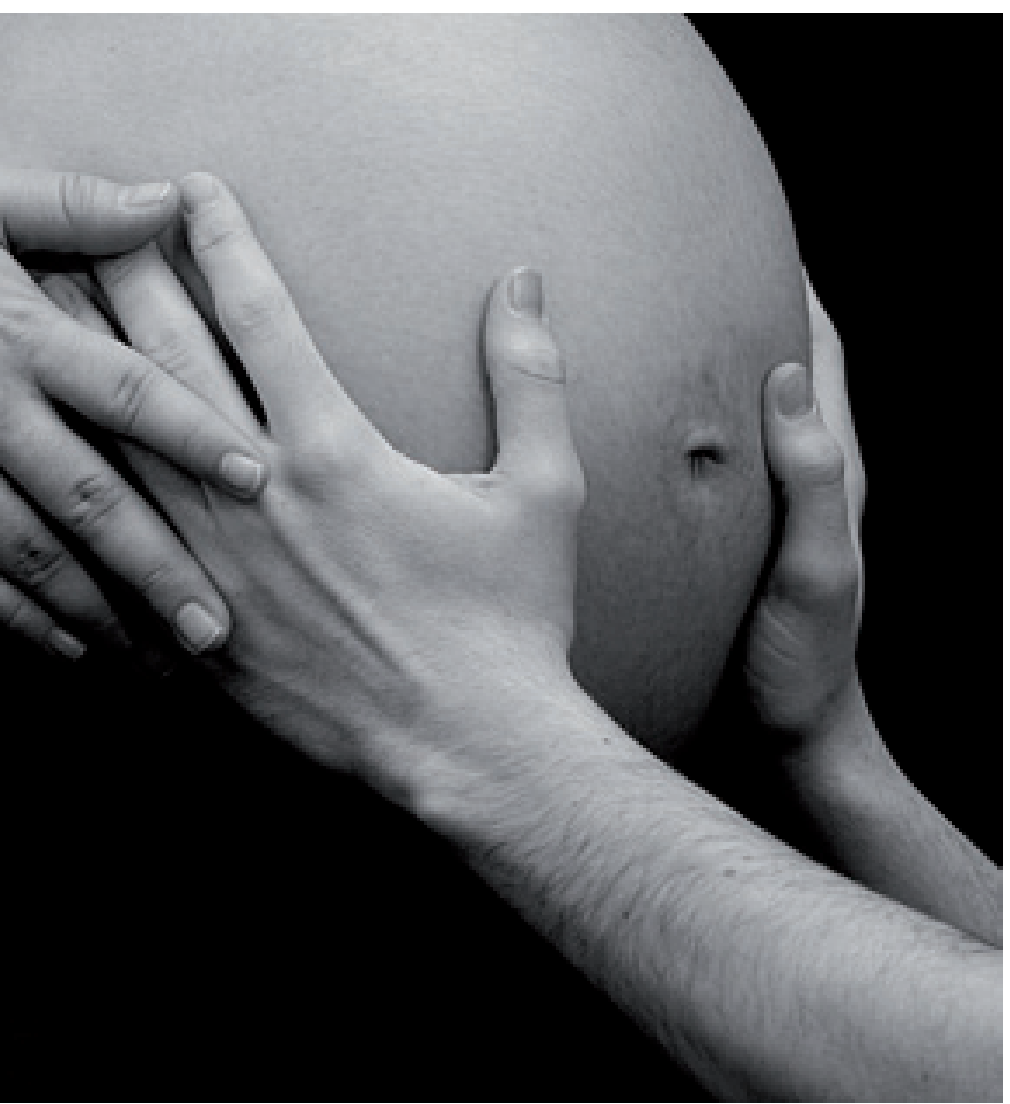

Las mujeres siguen llevando casi todo el peso de la vida familiar y doméstica: se encargan de las tareas del hogar y del cuidado de los hijos, mientras que los hombres colaboran desde un segundo plano.

realizar importantes aportaciones con las que dilucidar cómo la reproducción atraviesa las vidas de las mujeres. En lo que sigue, quisiéramos plantear, desde esta visión amplia y compleja de la reproducción, algunas ideas sobre cómo incide en las vidas de las mujeres.

\section{- LAS TRAMAS QUE DIBUJAN LA CENTRALIDAD DE LA REPRODUCCIÓN EN LAS MUJERES}

El análisis que ofrecemos es producto de datos procedentes de nuestra investigación, centrada en mujeres de nuestro contexto español actual -los primeros años del nuevo milenio-, si bien podrían encontrarse similitudes con mujeres de otros lugares y de otros momentos históricos.

Nuestro punto de partida descansa en la consideración de que todas las mujeres deben afrontar en diversos momentos de su vida la posibilidad de la reproducción, entendida en el triple sentido que planteábamos al principio: su dimensión biológica, de trabajo y de reproducción social. Veámoslo por partes.

En cuanto a la reproducción biológica, con todas las implicaciones que conlleva la procreación, encontramos el hecho de que las mujeres deben afrontar ineludiblemente la decisión de convertirse en madres o no. A este respecto, resulta clave el factor de la edad y, más en concreto, el límite de años superior que se contempla para la gestación, ya que los años de la mujer -ya sean 35, 40 o más- pueden considerarse como un marcador o señal de aviso que recuerda los riesgos que para ella puede suponer el embarazo y, por consiguiente, la necesidad de decidir sin más tardanza. Uno de los cambios más recientes es la consideración de la maternidad como una opción elegida; uno de los ejemplos más visibles lo constituyen las antes llamadas «madres solteras», que ahora han pasado a denominarse «madres por elección» (Rivas et al., 2011). Este cambio obedece a una importante transformación de las condiciones sociales, culturales y políticas que reconocen distintas formas de entender la maternidad, como diversas son las posibilidades de realizarse la procreación -no solo biológica-, las edades en las que acceder a ella, así como una considerable diversidad de modelos familiares. En consecuencia, hoy las mujeres disponen de más posibilidades de elegir con más libertad entre las diversas opciones de procreación biológica; si bien desde siempre han dispuesto de estrategias para elegir si ser madres o no, fueran estas visibles, reconocidas o sancionadas.

Aunque las mujeres decidan no procrear, el significado y las interpretaciones sociales de su corporalidad las siguen vinculando con esta posibilidad: la de ser madres, con las sucesivas implicaciones de embarazarse, parir y amamantar. Es reseñable que una decisión negativa en esto no clausura la significación social, las visiones y valores concernientes a sus cuerpos. Así, por ejemplo, fenómenos y dimensiones como la sexualidad, el ciclo menstrual, determinadas enfermedades como el cáncer de mama, o la menopausia, son leídos, por ellas mismas y por la sociedad en su conjunto, según unos presupuestos culturales que los evalúan bajo el criterio de la procreación o de la renuncia a ella: es decir, atendiendo al uso que se puede realizar o no de su cuerpo.

La segunda dimensión de la reproducción que señalábamos, la referente a perpetuar una forma de organización del trabajo, no ha sufrido cambios. Continúan siendo las mujeres las responsables fundamentales, casi siempre únicas, del trabajo doméstico o familiar. Los datos disponibles nos muestran que, a pesar de que las mujeres se han ido incorporando al trabajo salarial (y aun en esto sufriendo segregaciones y agravios comparativos), este hecho no ha tenido la contrapartida de que los hombres aumentasen su participación en el trabajo reproductivo. Las mujeres siguen siendo, especialmente en los momentos en que se desmantelan los estados del bienestar, como el actual, las principales suministradoras del trabajo doméstico, de cuidados y de crianza. Los datos muestran que existen en estas tareas algunas diferencias debidas a las diferencias de clase social, edad y etnia de las mujeres que las realizan; pero en ningún caso se observa la presencia de los varones (García Calvente 
et al., 2004). A este respecto, conviene apuntar que el perfil de la cuidadora es una mujer preferentemente soltera, para que su estado civil no interfiera en las tareas de crianza y cuidado. Por otro lado, la conciliación de la vida familiar y laboral origina la situación de «doble jornada» que sigue mostrando un rostro de mujer. Aun cuando algunas mujeres de hoy, que desempeñan una profesión y pertenecen a determinadas clases y grupos sociales, han sabido y han podido liberarse del mandato de la procreación, o la someten a una elección libre, este segundo mandato, el de la reproducción del trabajo, continúa cosido a sus vidas; así, se sentirán responsables del cuidado de otras personas o las harán responsables de ese cuidado de una u otra forma.

En relación a la tercera dimensión de la reproducción, la social o sistémica que es la que da la forma, organiza, dota de significado a las otras y configura el orden social, son indispensables las aportaciones de Gayle Rubin (1996). Esta autora subraya cómo estas dos dimensiones, las relativas a la procreación y a la división sexual del trabajo, y las consecuencias que necesariamente se derivan de ellas, han de ser ordenadas por un sistema de sexo/género. Es decir, la sociedad discrimina a sus individuos según la materia biológica del sexo y ordena la sexualidad, estableciendo un sistema de diferenciación neto. Este sistema se conforma dentro de unas condiciones políticas y económicas, que dotan de un sentido a los sexos, a la procreación y a la sexualidad, lo que constituye una verdadera economía política del sexo.

Las mujeres han interiorizado la importancia de respetar este orden social, lo defienden y lo transmiten. Paradójicamente, se descubre que el sistema ha depositado la responsabilidad de la reproducción de sí mismo en las mujeres. Con este objetivo, los mecanismos de control de las mujeres que se han constituido son amplios y diversos; pero todos tienen en común su intención de que se promueva y asegure la continuidad de este sistema de economía política del sexo. Adrianne Rich, en su estudio sobre la institución de la heterosexualidad, plantea que los medios para la dominación de las mujeres abarcan desde el acceso a la propiedad hasta el control de las conciencias y los cuerpos (1980; en Pichardo Galán, 2009). Por consiguiente, el control de los cuerpos de las mujeres, por ejemplo mediante el mantenimiento de determinadas visiones culturales -esencialistas, biologicistas, asociales, reduccionistas- acerca de la reproducción, estaría al servicio de la conservación y transmisión de este orden social. En nuestros trabajos de investigación del ámbito sanitario hemos mostrado cómo las mujeres son «domesticadas» a través de la imposición de ciertos patrones sobre sus cuerpos, con el fin de asegurar la procreación, la reproducción del orden laboral y, con ello, reproducir el sistema social en su conjunto (Montes, 2007). Por tanto,

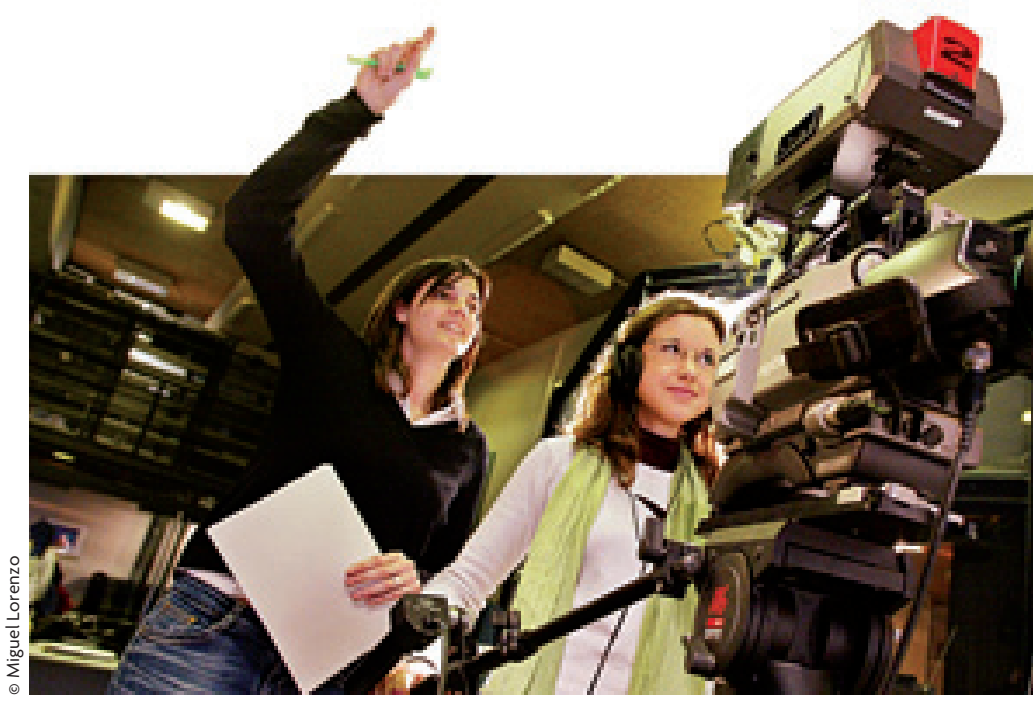

Cada vez más, las mujeres intentan conjugar sus propios intereses con los roles y patrones que la sociedad ha perpetuado en ellas durante años. Una lucha continua por mostrarse como mujeres completas y capaces, al margen de su tarea como madres.

\section{"NUESTRAS PROPIAS VIDAS E ITINERARIOS COMO MUJERES DE CIENCIA, AL IGUAL QUE TANTAS OTRAS, SE HAN VISTO CONFRONTADAS POR EL HECHO DE SER O NO MADRES"}
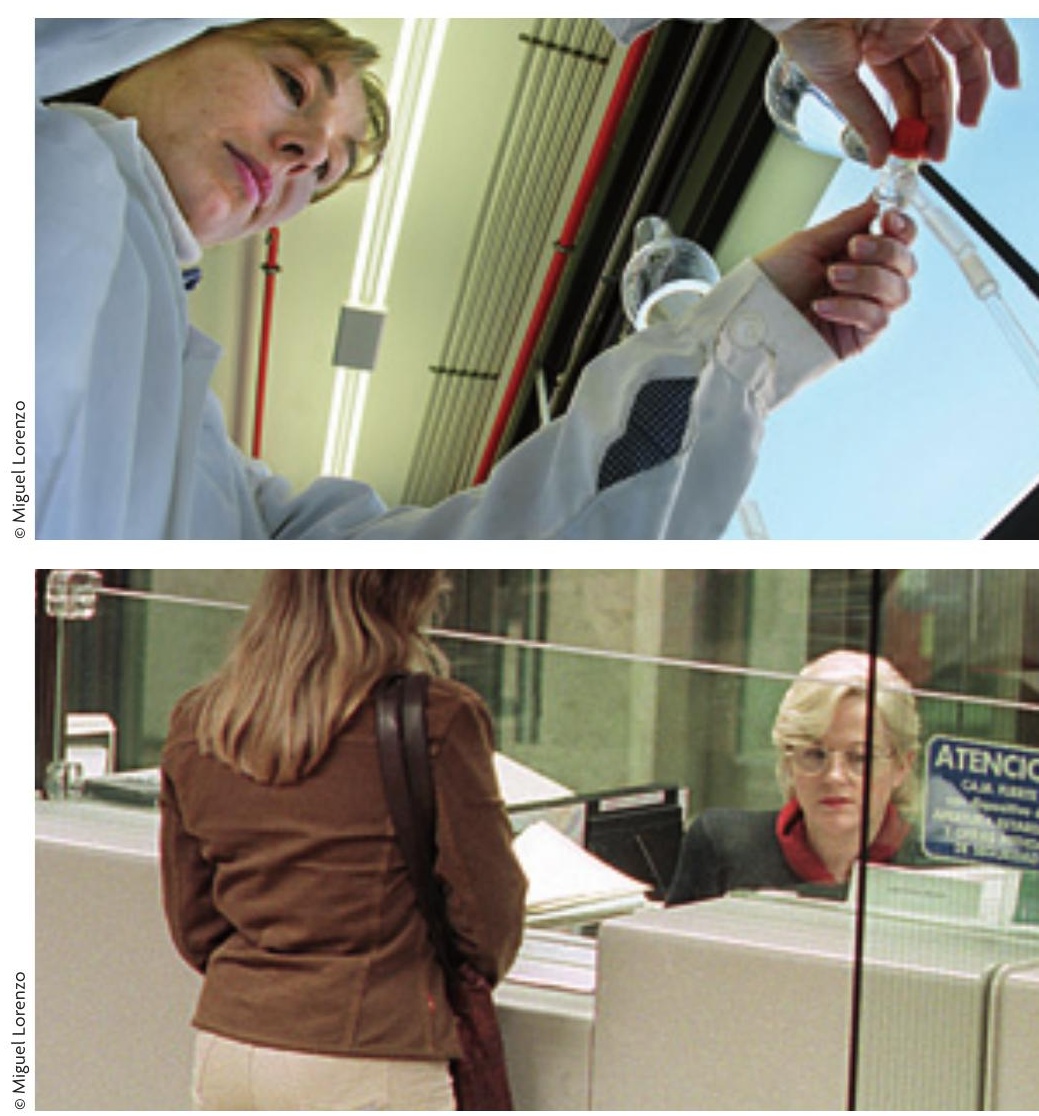


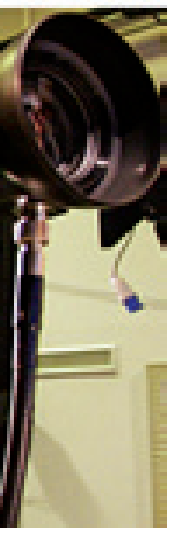

el sistema, desde esta configuración, necesita que la reproducción (en todas y cada una de sus dimensiones) siga ubicándose en el corazón de la vida de las mujeres.

En una sociedad postcapitalista como la nuestra, habría un guion establecido que marca que las vidas de las mujeres deben dirigirse a la reproducción. Esto sucede de manera inexorable, incluso aunque se den variaciones en este guion y las mujeres dispongan de diversas posiciones para contestar, afrontar y enfrentarse a, individualmente y como grupo, este asunto. Nuevamente, planteamos que si bien algunas mujeres han podido optar por «librarse» de la procreación biológica, del trabajo

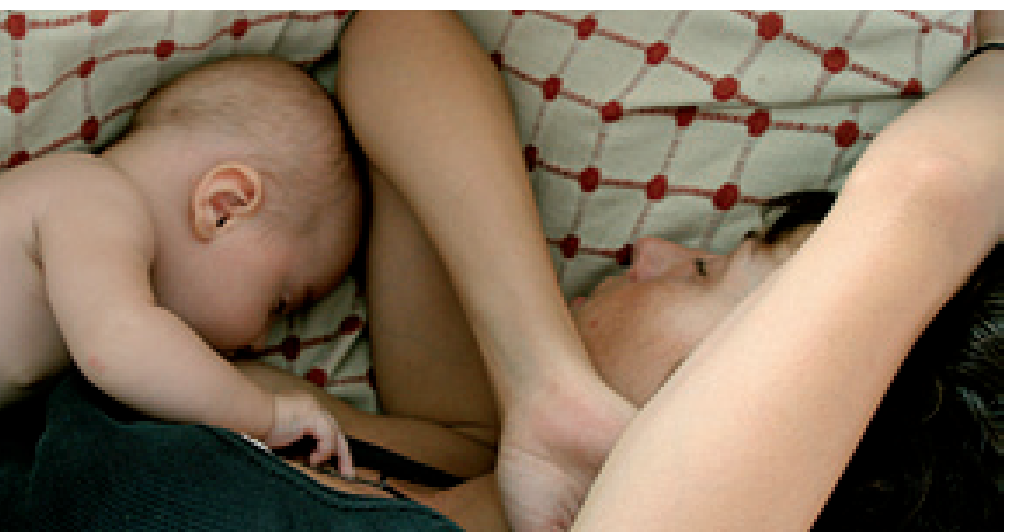

doméstico -en particular del cuidado de otros-, esto no ha supuesto el cambio del orden establecido. Sus acciones emancipadoras son necesarias, pero no suficientes para subvertir nuestro actual sistema de sexo/género. Sigue llamando la atención que a las mujeres en todas las facetas de su vida, incluso en el ámbito laboral, se las reclama para que se encarguen de la reproducción del grupo, y por extensión de lo afectivo, del cuidado y del mantenimiento de las relaciones sociales. Así, por ejemplo se describe la mentoría de las mujeres académicas como una forma de maternaje y cuidado, especialmente de aquellas que no hayan podido ser madres; mientras que en la mentoría de los hombres no se utilizan estas definiciones y modelos culturales que los vincularían con formas de la paternidad.

\section{CONCLUSIONES}

Hemos tratado de mostrar en este trabajo que, si manejamos un concepto amplio de reproducción como el que viene propuesto por la antropología, la reproducción resulta un área de la vida social unida de forma indisoluble -de momento- a las vidas de las mujeres; reproducción que cobra sentidos bien distintos en el caso de los hombres y que, si bien puede adoptar una amplia variedad, recae en inevitables monotonías.
La antropología nos insta a mirar cómo las personas construyen sus vidas y a comprender los engranajes de las mismas en el todo social. Desde esta perspectiva, nos ha impulsado a comprobar que no hay un carácter «natural» en la situación actual de las mujeres, sino que responde a un orden social, frente al cual muchas realizan un ejercicio de resistencia y lucha para conjugar sus intereses y sus proyectos dentro de la herencia sociocultural que han recibido. Así también, ahora en términos personales, nuestras propias vidas e itinerarios como mujeres de ciencia, al igual que tantas otras, se han visto confrontadas por el hecho de ser o no madres; $\mathrm{y}$, asimismo, por vernos instadas a encargarnos de la reproducción, de lo doméstico, de los cuidados. Somos plenamente conscientes de que las mujeres tienen que inventar y crear estrategias para amortiguar los costes y las consecuencias de la aceptación, pero sobre todo de la renuncia al cuidado, la maternidad y la crianza, destino al que se ven abocadas por el imperativo sociocultural.

Por consiguiente, queremos concluir afirmando que una de las claves para distinguir hoy a los hombres y las mujeres, para establecer la diferencia sexual, en nuestras sociedades, reside en que las mujeres son precisamente aquellas personas que están en este debate, en esta trama, en este desafío, interrogándose acerca de si ser madres, ser cuidadoras o responsables de la reproducción y sobre cómo hacerlo.

\section{BIBLIOGRAFÍA}

BlÁzQuez, M., 2005. «Aproximación a la Antropología de la Reproducción» Revista AIBR, 42: julio-agosto. Disponible en:

$<$ http://www.aibr.org/antropologia/42jul/articulos/jul0506.php >.

Browner, C. y C. Sargent, 1990. «Anthropology and Studies of Human Reproduction». In SArgent, C. y T. Johnson. Medical Anthropology: Contemporary Theory and Method. Praeger. Nueva York.

Comas, D., 1998. Antropología económica. Ariel. Barcelona.

Esteban, M. L.; Comelles, J. M. y C. Diez Mintegui, 2010. Antropología, género, salud y atención. Bellaterra. Barcelona.

Fons, V.; Piella, A. y M. VAldés, 2010. Procreación, crianza y género. Promociones y Publicaciones Universitarias. Barcelona.

García Calvente, M. M., Mateo Rodríguez, I. y Maroto-Navarro, G., 2004. «El impacto de cuidar en la salud y la calidad de vida de las mujeres». Gaceta Sanitaria, 18(2): 83-92.

Limón, A. y E. CAStellote, 1980. «La medicina popular en torno al embarazo y parto a principios de siglo». In M. Kenny y J. De Miguel. Antropología médica en España. Anagrama. Barcelona.

Montes, M. J., 2007. Las culturas del nacimiento: Representaciones y prácticas de las mujeres gestantes, comadronas y médicos. Tesis doctoral en Antropología Social y Cultural. Universidad Rovira i Virgili. Tarragona. Disponible en: <http://www.tdx.cat>.

Pichardo Galán, J. I., 2009. Relaciones homosexuales y nuevos modelos de familia. Bellaterra. Barcelona.

Rivas, A. M.; Jociles, M. I. y Moncó, B., 2011. «Las madres solteras por elección ¿ciudadana de primera y madres de segunda?». Revista Internacional de Sociología, 69(1): 121-142. DOI: 10.3989/ris.2009.06.27.

Rubin, G., 1996. «El tráfico de mujeres: notas sobre la "economía política" del sexo». In: LAmAs, M. (comp.). El género: la construcción cultural de la diferencia sexual. UNAM. México DF.

Maribel Blázquez Rodríguez. Profesora ayudante doctora del Departamento de Antropología Social. Universidad Complutense de Madrid.

María Jesús Montes Muñoz. Profesora titular del Departamento de Enfermería. Universidad Rovira i Virgili de Tarragona. 